\title{
Zinc supplementation inhibits the high glucose-induced EMT of peritoneal mesothelial cells by activating the Nrf2 antioxidant pathway
}

\author{
LILI GAO $^{1,2^{*}}$, YI FAN $^{1 *}$, XIULI ZHANG ${ }^{1}$, LINA YANG $^{1}$, WENYU HUANG $^{1}$, \\ TIANYU HANG ${ }^{1}$, MINGYANG LI ${ }^{1}$, SHUYAN DU ${ }^{1}$ and JIANFEI MA ${ }^{1}$ \\ ${ }^{1}$ Department of Nephrology, The First Affiliated Hospital, China Medical University, Shenyang, Liaoning 110001; \\ ${ }^{2}$ Department of Nephrology, The First Affiliated Hospital of Jinzhou Medical University, Jinzhou, Liaoning 121001, P.R. China
}

Received September 29, 2018; Accepted April 11, 2019

DOI: $10.3892 / \mathrm{mmr} .2019 .10260$

\begin{abstract}
The high glucose (HG)-induced epithelial-mesenchymal transition (EMT) of peritoneal mesothelial cells (PMCs) serves an important role in peritoneal fibrosis (PF) during peritoneal dialysis. Our previous study reported that zinc $(\mathrm{Zn})$ supplementation prevented the HG-induced EMT of rat PMCs in vitro. In the present study, the role of $\mathrm{Zn}$ in HG-induced EMT was investigated in vivo using a rat model of PF. Additionally, the molecular mechanisms underlying HG-induced EMT were studied in human PMCs (HPMCs). In the rat model of $\mathrm{PF}, \mathrm{HG}$ treatment increased the glucose transfer capacity and decreased the ultrafiltration volume. Histopathological analysis revealed peritoneal thickening, increased expression of vimentin and decreased expression of E-cadherin. $\mathrm{ZnSO}_{4}$ significantly ameliorated the aforementioned changes, whereas $\mathrm{Zn}$ inhibition by clioquinol significantly aggravated the effects of HG on rats. The effects of $\mathrm{Zn}$ on HPMCs was assessed using western blot analysis, Transwell assays and flow cytometry. It was revealed that $\mathrm{Zn}$ also significantly suppressed the extent of the EMT, and reduced reactive oxygen species production and the migratory ability of HG-induced HPMCs, whereas Zn inhibition by $\mathrm{N}^{\prime}, \mathrm{N}^{\prime}, \mathrm{N}^{\prime}, \mathrm{N}^{\prime}$-tetrakis (2-pyridylmethyl) ethylenediamine significantly potentiated the HG-induced EMT of HPMCs. HG-stimulated HPMCs exhibited increased expression of nuclear factor-like 2 (Nrf2) in the nucleus, and total cellular NAD $(\mathrm{P}) \mathrm{H}$ quinone dehydrogenase 1 (NQO1) and heme oxygenase-1 (HO-1), the target proteins of the Nrf2 antioxidant pathway. Zn supplementation further promoted nuclear Nrf2
\end{abstract}

Correspondence to: Dr Jianfei Ma, Department of Nephrology, The First Affiliated Hospital, China Medical University, 155 Nanjing North Street, Shenyang, Liaoning 110001, P.R. China

E-mail: majianfei56@sohu.com

*Contributed equally

Key words: high glucose, zinc, epithelial-mesenchymal transition, mesothelial cells, nuclear factor-like 2 antioxidant pathway expression, and increased the expression of target proteins of the Nrf2 antioxidant pathway, whereas $\mathrm{Zn}$ depletion decreased nuclear Nrf2, NQO1 and HO-1 expression compared with the HG group. In conclusion, $\mathrm{Zn}$ supplementation was proposed to suppress the effects of HG on the EMT by stimulating the Nrf2 antioxidant pathway and subsequently reducing oxidative stress in PMCs.

\section{Introduction}

Peritoneal dialysis (PD) is one of the most common treatments for managing patients with end-stage renal disease (ESRD). On average, $\sim 11 \%$ of patients with ESRD receive PD globally (1); however, PD solution can induce pathological changes in the peritoneum with regards to function and structure, such as peritoneal fibrosis (PF), which may result in the failure of PD $(2,3)$. These changes occur due to the high glucose concentrations (HG), products of glucose degradation, lactate and low $\mathrm{pH}$ of PD solution (4).

Accumulating evidence indicates that the epithelial-mesenchymal transition (EMT) is a pivotal point in the progression and pathogenesis of peritoneal fibrosis $(\mathrm{PF})$, resulting in the loss of epithelial characteristics and gain of mesenchymal characteristics (5-7). Previous studies reported that $\mathrm{HG}$ increased the expression of transforming growth factor- $\beta 1$ (TGF- $\beta 1$ ), which induced the EMT in peritoneal mesothelial cells (PMCs) $(8,9)$. Additionally, it has been reported that HG-based PD solution induced peritoneal EMT by generating reactive oxygen species (ROS), whereas antioxidants effectively inhibited the $\mathrm{HG}$-induced EMT in the rat peritoneum and human PMCs (HPMCs) $(10,11)$.

Zinc $(\mathrm{Zn})$ is an essential microelement that is involved in various cellular functions, including the transcription and replication of genes, signal transduction, and the regulation of cell apoptosis and differentiation (12-15). Previous studies demonstrated that $\mathrm{Zn}$ supplementation inhibited fibrosis in various animal models, including models of myocardial fibrosis, liver fibrosis, perivascular fibrosis, vasculitis and cystic fibrosis (16-19). Our previous study reported that $\mathrm{Zn}$ serves an important role in preventing the HG-induced EMT of rat PMCs in vitro (20); however, its functions and mechanisms 
in vivo remain unclear. In the present study, the role of $\mathrm{Zn}$ in the HG-induced EMT was investigated in a rat model of PF in vivo, and the effects of $\mathrm{Zn}$ on HPMCs were determined, in addition to the underlying molecular mechanisms.

\section{Materials and methods}

Main reagents. $\mathrm{ZnSO}_{4}$ (Xinhua Pure Chemical Industries), 4.25\% dextrose PD fluid (Baxter Healthcare Ltd.), Zn chelator $\mathrm{N}, \mathrm{N}, \mathrm{N}^{\prime}, \mathrm{N}^{\prime}$-tetrakis (2-pyridylmethyl) ethylenediamine (TPEN; Sigma-Aldrich; Merck KGaA) and clioquinol (CQ; Sigma-Aldrich; Merck KGaA) were used during the study. Monoclonal antibodies against E-cadherin (cat. no. 3195), vimentin (cat. no. 5741), NAD(P)H quinone dehydrogenase 1 [cat. no. 3187, (NQO-1)], heme oxygenase-1 [cat. no. 5853, (HO-1)], Nrf-2 (cat. no. 12721), GAPDH (cat. no. 5174) and Lamin B (cat. no. 13435) were purchased from Cell Signaling Technology, Inc. A Pierce ECL detection kit was obtained from Pierce (Thermo Fisher Scientific, Inc.). All reagents used were of analytical grade.

Animal model of PF. A total of 40 male Sprague-Dawley rats (200-250 g, 8-10 weeks) were obtained from the Center of Experimental Animals of China Medical University (Shenyang, China). Rats were housed in controlled conditions, at a temperature of $20-25^{\circ} \mathrm{C}$ with $40-70 \%$ humidity under a 12:12-h light/dark cycle, with access to food and water ad libitum.

Rats were randomly allocated into four groups with 10 rats in each group: The negative control (NC) group, which were treated daily via intraperitoneal administration of $100 \mathrm{ml} / \mathrm{kg}$ of $0.9 \%$ saline for 4 weeks; the HG group, which received daily intraperitoneal administration of $100 \mathrm{ml} / \mathrm{kg}$ of $4.25 \%$ dextrose $\mathrm{PD}$ fluid for 4 weeks; the $\mathrm{HG}+\mathrm{Zn}$ group, which received daily intraperitoneal administration of $3 \mathrm{mg} / \mathrm{kg} \mathrm{ZnSO}_{4}$ and $100 \mathrm{ml} / \mathrm{kg}$ of $4.25 \%$ dextrose PD fluid for 4 weeks; the $\mathrm{HG}+\mathrm{CQ}$ group, which were treated daily via intraperitoneal administration of $20 \mathrm{mg} / \mathrm{kg} \mathrm{CQ}$ and $100 \mathrm{ml} / \mathrm{kg}$ of $4.25 \%$ dextrose PD fluid for 4 weeks. Prior to sacrifice, $100 \mathrm{ml} / \mathrm{kg}$ of $4.25 \%$ dextrose PD fluid was intraperitoneally administered to the rats. At $2 \mathrm{~h}$ later, the rats were anaesthetized, the peritonea were opened, and all the fluid contents were immediately collected to determine the volume (ml). The ultrafiltration volume (UV) was calculated using the following formula: Drainage volume-initial injection volume. The glucose concentration in the dialysate was determined using a glucose assay kit (cat. no. ab65333, Abcam) to assess the transfer capacity of glucose, according to the manufacture's protocols. In addition, the visceral and parietal peritonea were kept for subsequent examination.

All animal experiments in the present study were approved by the Institutional Animal Ethics Committee of China Medical University. All animal procedures were performed according to the Animal Care Institutional Guidelines for experimental animals.

Pathology and immunohistochemistry. For pathological examination, the parietal peritoneum was fixed in $4 \%$ paraformaldehyde for $12 \mathrm{~h}$ at room temperature. Paraffin-embedded sections $(4 \mu \mathrm{m})$ were prepared and stained with hematoxylin (room temperature for $15 \mathrm{~min}$ ) and eosin (room temperature for $3 \mathrm{~min}$; H\&E) and/or Masson's trichrome (room temperature for $10 \mathrm{~min}$ ); 5 random visual fields from each section were imaged under a light microscope (magnification, x10 and 20) and selected for measuring the thickness of the peritoneum. Image-Pro Plus version 6.0 (Media Cybernetics, Inc.) was used to analyze the images. 'Peritoneal thickness', which indicated the extent of fibrosis, was defined as the thickness of the submesothelial zone measured from the inner surface of the abdominal muscle to the mesothelium (21). The average of five random visual fields was used to quantify the thickness of each section.

For immunohistochemical staining, paraffin-embedded sections were heated at $60^{\circ} \mathrm{C}$ for $10 \mathrm{~min}$, washed with xylene and rehydrated in a descending alcohol series. The deparaffinized sections were blocked with $3 \%$ hydrogen peroxide (room temperature for $10 \mathrm{~min}$ ) and non-immune goat serum (KIT-9710; Fuzhou Maixin Biotech Co., Ltd.; room temperature for $20 \mathrm{~min}$ ). Then, tissues were incubated with primary antibodies against E-cadherin (1:200; Cell Signaling Technology, Inc.) and vimentin (1:200; Cell Signaling Technology, Inc.) at $4^{\circ} \mathrm{C}$ overnight following antigen retrieval in boiling sodium citrate buffer (10 mM, pH 6.0) for $5 \mathrm{~min}$. After washing with phosphate-buffered saline (PBS), tissue sections were incubated with a horseradish peroxidase (HRP)-conjugated secondary antibody (KIT-9710; Fuzhou Maixin Biotech Co., Ltd.) at $37^{\circ} \mathrm{C}$ for $30 \mathrm{~min}$. Reaction products were visualized by incubation with 3,3'-diaminobenzidine at $37^{\circ} \mathrm{C}$ for $30 \mathrm{~min}$ and then counterstained with hematoxylin at room temperature for $3 \mathrm{~min}$. As a negative control, tissue samples were subject to the same staining procedures with no incubation with primary antibodies. A minimum of five fields were randomly selected in each section and images were acquired by a fluorescence microscope (Nikon Corporation) at a magnification of $\mathrm{x} 20$. Image-Pro Plus was used to calculate the number of cells positive for E-cadherin and vimentin in each field. According to the proportion of positive cells, the following standard was used: 0 , negative; $1,1-20 \% ; 2,21-50 \% ; 3,51-80 \%$; 4, 81-100\%.

Cell culture. HMrSV5, the HPMC line, was provided by Professor Huimian $\mathrm{Xu}$ of The First Affiliated Hospital of China Medical University (Shenyang, China). Cells were isolated from human omentum by enzymatic disaggregation and cultured in RPMI-1640 medium (Gibco; Thermo Fisher Scientific, Inc.) supplemented with $10 \%$ FBS (Gibco; Thermo Fisher Scientific, Inc.) (22). All cells were incubated at $37^{\circ} \mathrm{C}$ in a $5 \% \mathrm{CO}_{2}$. atmosphere. Confluent cells presented as a monolayer with characteristic cobblestone-like appearance. The following groups were allocated: The NC group (HPMCs without any treatment); the HG group [HPMCs treated with $\mathrm{HG}(126 \mathrm{mM})]$; the $\mathrm{HG}+\mathrm{Zn}$ group [HPMCs co-treated with $\mathrm{HG}(126 \mathrm{mM})$ and $\left.\mathrm{ZnSO}_{4}(10 \mu \mathrm{M})\right]$; the $\mathrm{HG}+$ TPEN group [HPMCs co-treated with HG (126 mM) and TPEN $(1 \mu \mathrm{M})$ ]; and the $\mathrm{Zn}$ group [HPMCs treated with $\mathrm{ZnSO}_{4}(10 \mu \mathrm{M})$ alone]. Cultures were incubated for $48 \mathrm{~h}$.

Cell migration assay. Cell migration ability was assessed using a Transwell assay with 24-well Transwell chambers (Corning, Inc.) with $8-\mu \mathrm{m}$ pores. A cell suspension containing $5 \times 10^{5}$ cells $/ \mathrm{ml}$ was seeded into the upper chamber in serum-free 
medium. The medium in the lower chamber contained $10 \%$ FBS. Cells were untreated or pre-treated with $\mathrm{ZnSO}_{4}(10 \mu \mathrm{M})$ or TPEN $(1 \mu \mathrm{M})$ in the presence or absence of HG $(126 \mathrm{mM})$. Following incubation at $37^{\circ} \mathrm{C}$ for $24 \mathrm{~h}$, a cotton-tipped swab was used to carefully remove the cells that had not migrated through the pores; the cells that had migrated onto the lower surface were stained with $0.1 \%$ crystal violet for $5 \mathrm{~min}$ at room temperature. Five random visual fields in each section were analyzed under a light microscope (magnification, x20) and cells were counted. Three independent experiments were performed.

Detection of intracellular ROS levels. An ROS assay was performed using an ROS assay kit (Beyotime Institute of Biotechnology) according to the manufacturer's protocols. Briefly, HPMCs were treated as aforementioned. Then, $5 \times 10^{6}$ cells were incubated with $10 \mu \mathrm{mol} / 1$ dichlorodihydrofluorescein diacetate (DCFH-DA; Beyotime Institute of Biotechnology) probes at $37^{\circ} \mathrm{C}$ for $30 \mathrm{~min}$. Following washing with PBS three times, DCFH-DA was deacetylated by a intracellular nonspecific esterase to 2,7-dichlorofluorescein (DCF), a fluorescent compound. The fluorescence spectrum of DCF which is excited at $488 \mathrm{~nm}$ and emitted at $525 \mathrm{~nm}$ is very similar to FITC. So FITC can be used to detect DCF fluorescence using a flow cytometer (BD FACSCaliber; BD Biosciences). Results were analyzed using Cell Quest software version 5.1 (BD Biosciences).

Western blotting. For western blotting, tissues and HPMCs were lysed in RIPA buffer (Sigma-Aldrich; Merck KGaA) with protease inhibitors (Sigma-Aldrich; Merck KGaA). Supernatants containing total proteins were harvested to detect HO-1, NQO1, E-cadherin, vimentin and GAPDH. Nuclear proteins of the HPMCs were extracted using a nuclear protein extraction kit (Sigma-Aldrich; Merck KGaA) to detect Lamin B and nuclear factor-like 2 (Nrf2). Protein $(30 \mu \mathrm{g})$ determined by BCA assay (Beyotime Institute of Biotechnology) was separated via 10\% SDS-PAGE and transferred to polyvinylidene difluoride membranes, which were blocked with 5\% BSA (Beijing Solarbio Science \& Technology Co., Ltd.) in Tris-Buffered saline containing 0.1\% Tween-20 for $1 \mathrm{~h}$ at room temperature, and then incubated with specific primary antibodies (1:1,000; Cell Signaling Technology, Inc.) against HO-1, NQO, GAPDH, E-cadherin, vimentin, Nrf2 and Lamin $\mathrm{B}$ at $4^{\circ} \mathrm{C}$ overnight. Blots were subsequently incubated with a horseradish peroxidase-conjugated secondary antibody (cat. no. E030120-02; 1:2,000; EarthOx Life Sciences) for $1 \mathrm{~h}$ at room temperature. Proteins were visualized using chemiluminescence and clarity western ECL substrate (Bio-Rad Laboratories, Inc.). Densitometric analysis of the western blots was performed using ImageJ v1.48 software (National Institutes of Health).

Statistical analysis. SPSS (Version 17.0; SPSS, Inc.) was used to analyze statistics. All data were presented as the mean \pm standard deviation, and all experiments were performed at least three times. After statistical significance was established by one-way ANOVA, post hoc multiple comparisons were performed using Tukey's multiple comparison test. $\mathrm{P}<0.05$ was considered to indicate a statistically significant difference.

\section{Results}

Zn prevents fibrosis in a rat model of $P F$. As presented in Fig. 1, the rats in the $\mathrm{HG}$ group exhibited significantly reduced UV values and an increased glucose transfer capacity compared with the NC. Zn supplementation significantly reduced the glucose transfer capacity and increased the UV compared with HG treatment alone, whereas CQ treatment significantly increased the glucose transfer capacity, but decreased the UV.

It was observed via $H \& E$ staining that inflammatory cells infiltrated the interstitium, fibers were exposed, mesothelial cells became round and cylindrical, and cells shed in the HG group (Fig. 2A and B). Furthermore, the HG + CQ group presented a further elevated inflammatory response and significantly thicker peritoneum compared with the HG group. Masson's trichrome staining also demonstrated that HG treatment significantly increased the thickness of the peritoneal collagen fibers compared with the NC group (Fig. 2C and D). This thickening may increase the permeability of peritoneal membrane and the absorption of glucose, which could reduce the osmotic pressure of peritoneal permeation and affect ultrafiltration. Immunohistochemical staining revealed significantly increased expression of vimentin, and decreased expression of E-cadherin in the HG group, compared with the NC group (Fig. 3). Zn supplementation significantly ameliorated these pathological alterations, whereas CQ aggravated the effects of HG on the PMCs (Figs. 2 and 3).

Zn inhibits the HG-induced EMT of HPMCs. Concentrations and durations of treatment of $\mathrm{HG}, \mathrm{Zn}$ and TPEN were established according to our previous studies $(20,23,24)$. Following exposure of HPMCs to HG for $48 \mathrm{~h}$, E-cadherin expression decreased, whereas the expression of vimentin was significantly upregulated compared with the NC group (Fig. 4). Co-treatment with $\mathrm{Zn}(10 \mu \mathrm{M})$ for $48 \mathrm{~h}$ in HPMCs resulted in a significant alleviation of HG-induced EMT-associated alterations, whereas co-treatment with the $\mathrm{Zn}$ chelator TPEN $(1 \mu \mathrm{M})$ significantly aggravated HG-induced changes in expression, consistent with the results of the in vivo experiments.

Zn inhibits the HG-induced migration of HPMCs. The roles of $\mathrm{Zn}$ in the migration of HPMCs were investigated by using a Transwell cell migration assay; cells exhibit increased migration following EMT $(25,26)$. HG stimulation for $24 \mathrm{~h}$ significantly increased the migration of HPMCs compared with the $\mathrm{NC}$ group (Fig. 5). Co-treatment with $\mathrm{ZnSO}_{4}$ significantly reversed the HG-stimulated increase in migration, whereas co-treatment with the $\mathrm{Zn}$ chelator TPEN further promoted the migration of HPMCs (Fig. 5).

Zn inhibits $H G$-induced ROS generation of HPMCs. The effects of $\mathrm{Zn}$ on the intracellular generation of ROS were also determined, as ROS generation may promote the onset of EMT. HG significantly increased the levels of ROS in HPMCs compared with the NC group (Fig. 6). Co-treatment with $\mathrm{ZnSO}_{4}$ significantly inhibited the HG-stimulated increase in ROS levels, whereas co-treatment with the $\mathrm{Zn}$ chelator TPEN further increased ROS levels in HPMCs (Fig. 6). 

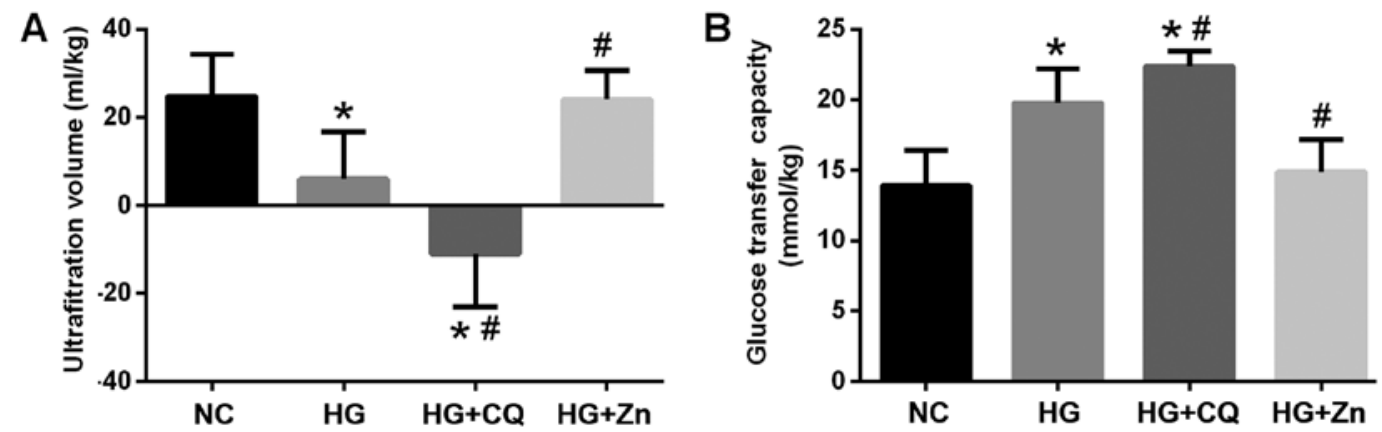

Figure 1. Effects of $\mathrm{Zn}$ on peritoneal function in a rat model of peritoneal fibrosis. (A) Ultrafiltration volume and (B) glucose transfer capacity were used to indicate the peritoneal function in each group. Data are presented as the mean \pm standard deviation. P-values were determined using ANOVA with Tukey's post hoc test. ${ }^{\text {P }}<0.05$ vs. NC; ${ }^{~} \mathrm{P}<0.05$ vs. HG. NC, negative control; $\mathrm{HG}$, high glucose; $\mathrm{CQ}$, chloroquine; $\mathrm{Zn}, \mathrm{ZnSO}_{4}$.

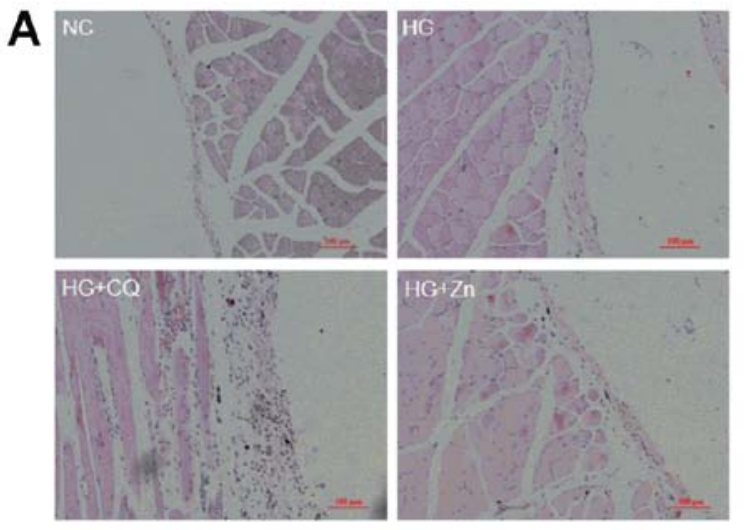

B

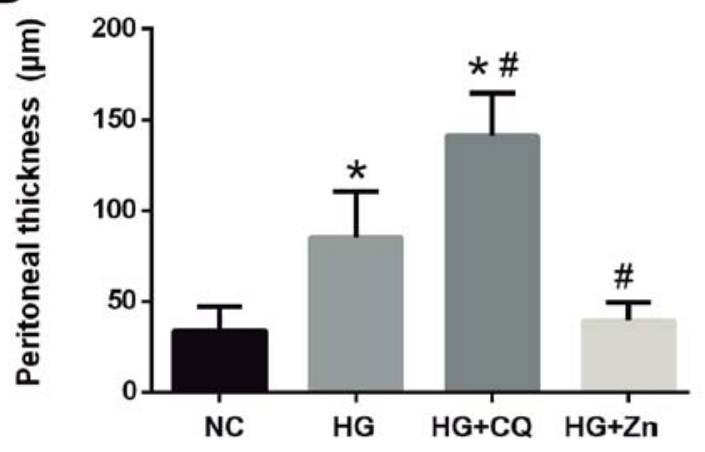

C

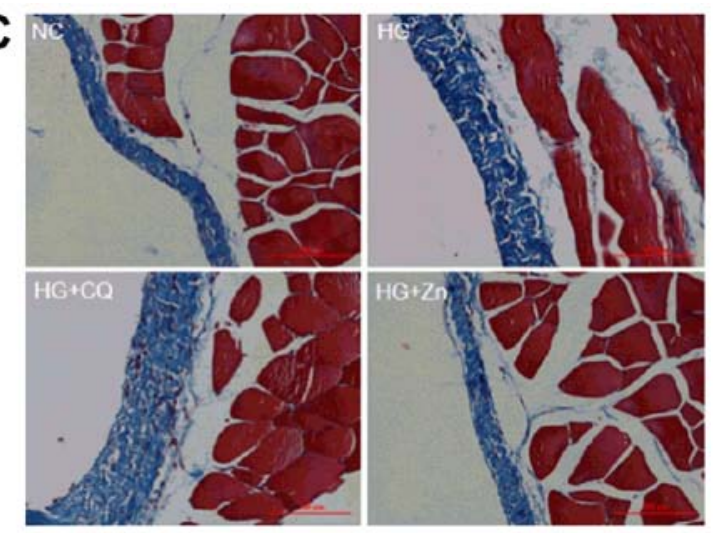

D

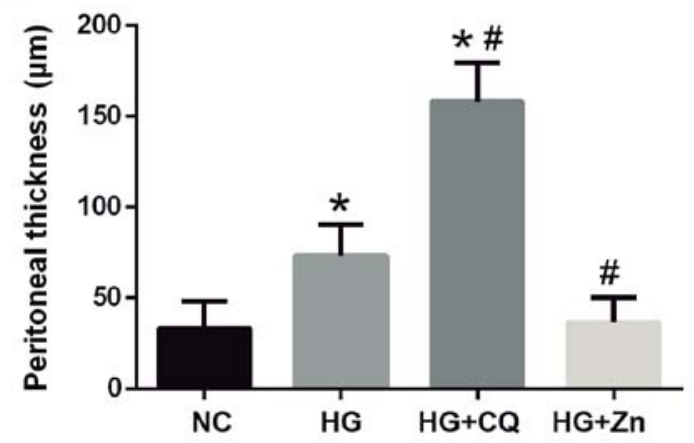

Figure 2. Effects of $\mathrm{Zn}$ on the peritoneal thickness in a rat model of peritoneal fibrosis. (A) H\&E staining of the peritoneum and (B) peritoneal thickness in rats from each group (magnification, x10). (C) Masson's trichrome staining of the peritoneum and (D) peritoneal thickness in rats from each group (magnification, $\mathrm{x} 20$ ). Peritoneal thickness was defined as the thickness of the submesothelial zone measured from the inner surface of the abdominal muscle to the mesothelium. Data are presented as the mean \pm standard deviation. P-values were determined using ANOVA with Tukey's post hoc test. * $<0.05$ vs. NC; ${ }^{\#} \mathrm{P}<0.05$ vs. HG. NC, negative control; $\mathrm{HG}$, high glucose; $\mathrm{CQ}$, chloroquine; $\mathrm{Zn}, \mathrm{ZnSO}_{4}$.

Zn activates the Nrf2 antioxidant pathway of HPMCs induced by $H G$. The Nrf2 antioxidant pathway has been reported to be involved in regulating ROS generation and the EMT $(27,28)$. Therefore, the effects of $\mathrm{Zn}$ on the activity of the Nrf 2 antioxidant pathway in HPMCs was determined via western blotting. As presented in Fig. 7, the expression levels of nuclear Nrf2 and target proteins of the Nrf2 antioxidant pathway, including NQO1 and HO-1, were upregulated in HG-induced HPMCs compared with the NC group. Co-treatment with TPEN $(1 \mu \mathrm{M})$ notably inhibited the expression of these proteins, whereas co-treatment with $\mathrm{ZnSO}_{4}(10 \mu \mathrm{M})$ significantly promoted the expression of nuclear Nrf2, NQO1 and HO-1 compared with the HG group. The group treated with $\mathrm{Zn}$ alone presented no significant alterations in expression compared with the NC group.

\section{Discussion}

In the present study, a rat model of PF was induced by a $4.25 \%$ glucose dialysis solution. The HG group exhibited the induction of EMT and characteristics of PF compared with the control group, indicating that this model is a reliable animal model for studying PD-induced PF. Zn deletion employed by CQ further promoted HG-induced EMT in the rat PF model. Notably, Zn supplementation was able to significantly inhibit the EMT by promoting the expression of E-cadherin, inhibiting the 
A

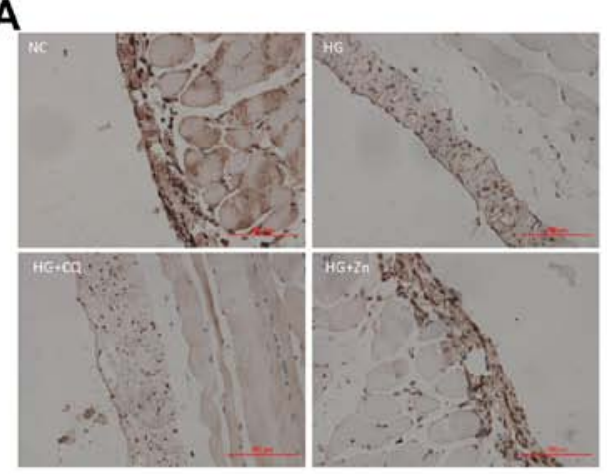

B

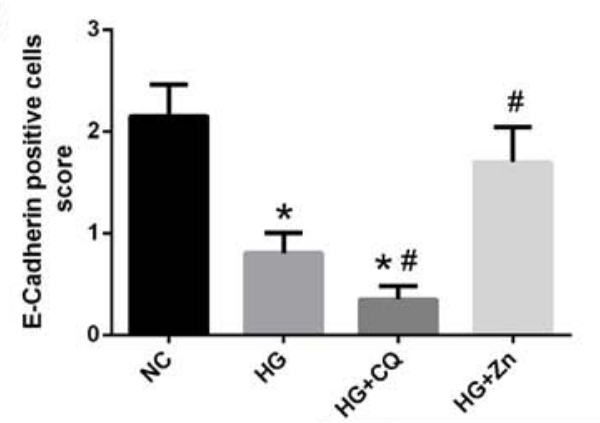

C

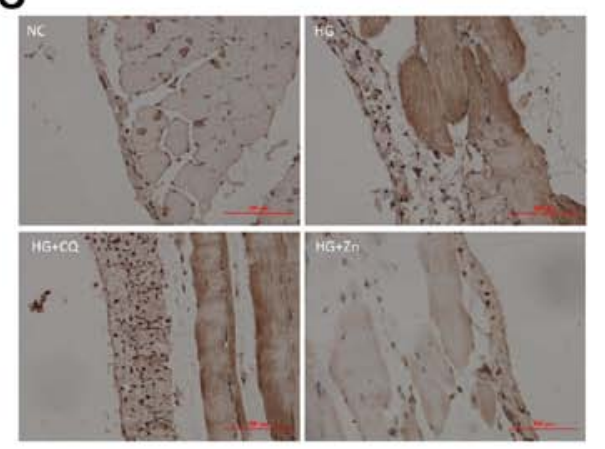

D

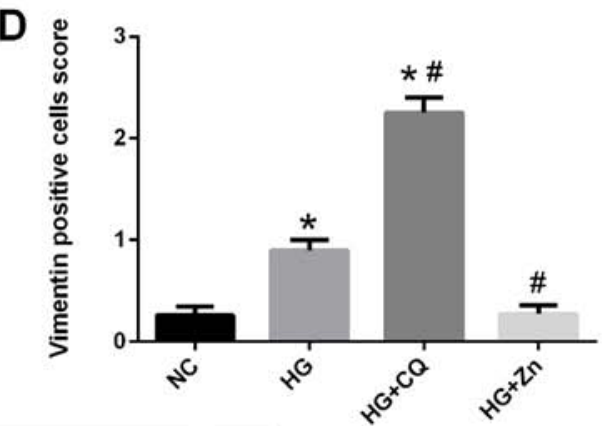

E

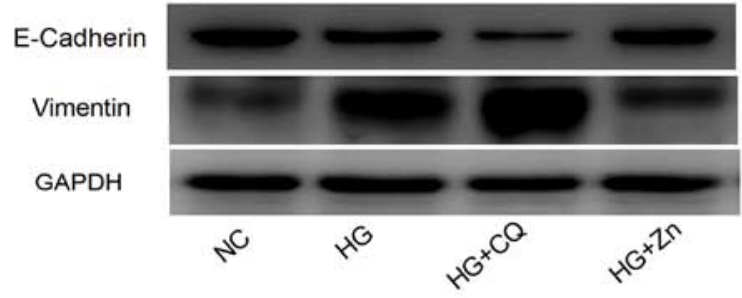

$\mathbf{F}$

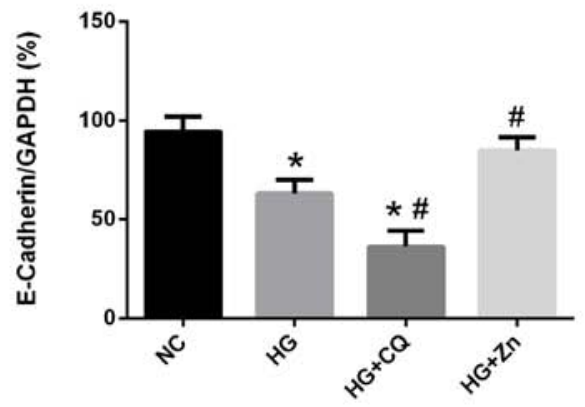

G

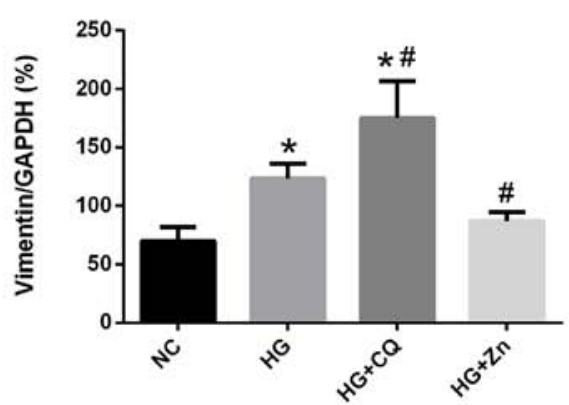

Figure 3. Effects of $\mathrm{Zn}$ on the expression of E-cadherin and vimentin in the peritoneum in a rat model of peritoneal fibrosis. Expression of (A and B) E-cadherin and $(\mathrm{C}$ and $\mathrm{D})$ vimentin as determined using immunohistochemistry (magnification, $\mathrm{x} 20$ ). (E) Western blotting assay of E-cadherin and vimentin in the peritoneum of rats from each group. ( F and G) Densitometry analysis. Data are presented as the mean \pm standard deviation. P-values were determined using ANOVA with Tukey's post hoc test. " $\mathrm{P}<0.05$ vs. NC; ${ }^{~} \mathrm{P}<0.05$ vs. HG. NC, negative control; $\mathrm{HG}$, high glucose; CQ, chloroquine; Zn, ZnSO ${ }_{4}$.

expression of vimentin and decreasing the peritoneum thickness, thus improving peritoneal function. Various studies $(29,30)$ have demonstrated the capacity of CQ to target chelatable $\mathrm{Zn}$. There is evidence that mice injected intraperitoneally with $\mathrm{CQ}$ exhibited notable reductions in chelatable $\mathrm{Zn}$ in the brain, testes and pancreas $(31,32)$. CQ has also been reported to be a therapeutic agent due to its ability to reduce $\mathrm{Zn}$ accumulation in neuritic plaques of Alzheimer's disease (33). Intraperitoneally injected CQ is considered safe (32). The lipophilic nature of CQ suggests that it can pass directly into the peritoneum (32). Therefore, CQ was selected in the present study for the in vivo experiments to inhibit the effects of endogenous $\mathrm{Zn}$.

TPEN $\left[K_{d}=2.6 \times 10^{-16} \mathrm{M}(34)\right]$, another $\mathrm{Zn}$ chelator, exhibited an increased affinity for $\mathrm{Zn}^{2+}$ compared with $\mathrm{CQ}$
$\left[\mathrm{K}_{\mathrm{d}}=\sim 1 \times 10^{-7} \mathrm{M}(34)\right]$. Previous studies $(35,36)$ indicated TPEN was an effective tool for inhibiting $\mathrm{Zn}$ in cell models, so it was selected for the in vitro experiments.

A concentration of $126 \mathrm{mM}$ glucose was used to induce EMT, based on our previous in vitro study $(20,23)$. EMT not only serves an important role in certain physiological processes, such as embryogenesis, but also is involved in the pathophysiological process of tissue fibrosis (37).

The EMT of PMCs has been reported to be a crucial mechanism underlying PF, and increasing evidence has indicated that inhibition of the EMT may alleviate PF $(38,39)$. Numerous genes are involved in regulating EMT, including E-cadherin, occludin, desmoplakin, $\alpha$-smooth muscle actin, $\mathrm{N}$-cadherin, vimentin, fibronectin, collagen I and snail $(40,41)$. In the 
A
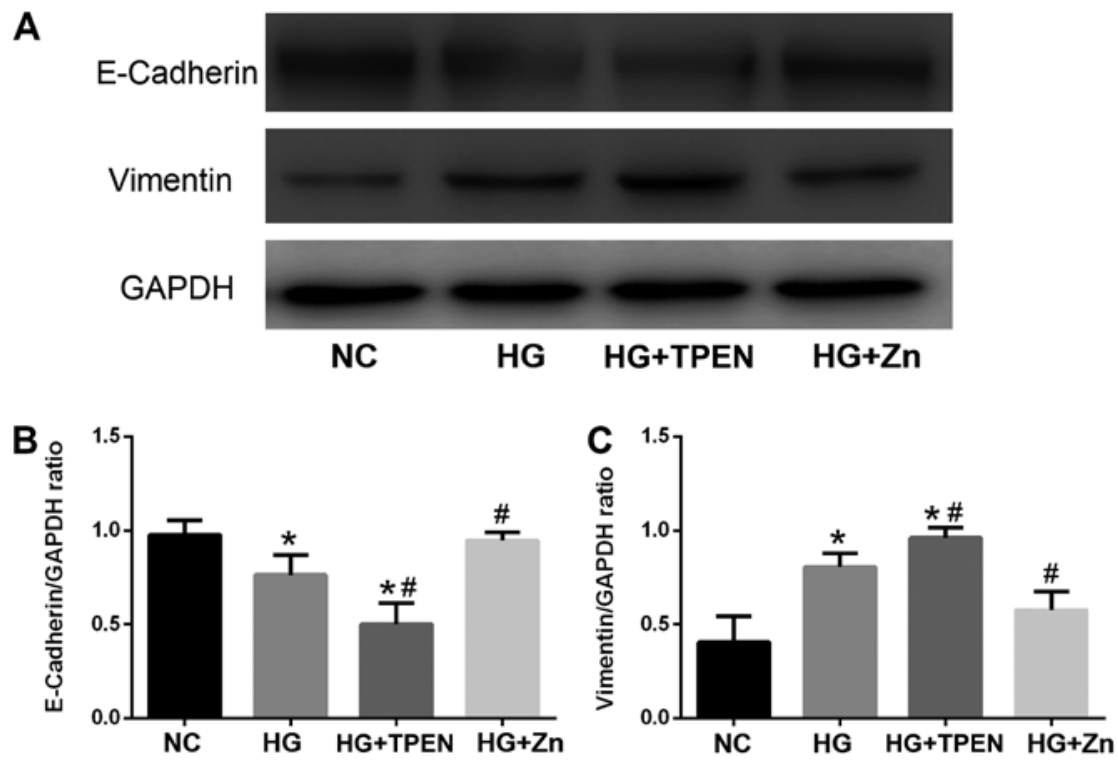

Figure 4. Effects of Zn on the HG-induced epithelial-mesenchymal transition of HPMCs. (A) Western blotting. Densitometry analysis of (B) E-cadherin and (C) vimentin expression in HPMCs following co-treatment with $\mathrm{HG}$, and TPEN or $\mathrm{ZnSO}_{4}$ for $48 \mathrm{~h}$. Data are presented as the mean \pm standard deviation. P-values were determined using ANOVA with Tukey's post hoc test. " $\mathrm{P}<0.05$ vs. NC; ${ }^{\text {"P}} \mathrm{P}<0.05$ vs. HG. NC, negative control; HG, high glucose; TPEN, N,N,N',N'-tetrakis (2-pyridylmethyl) ethylenediamine; $\mathrm{Zn}, \mathrm{ZnSO}_{4}$; HPMC, human peritoneal mesothelial cell.

A

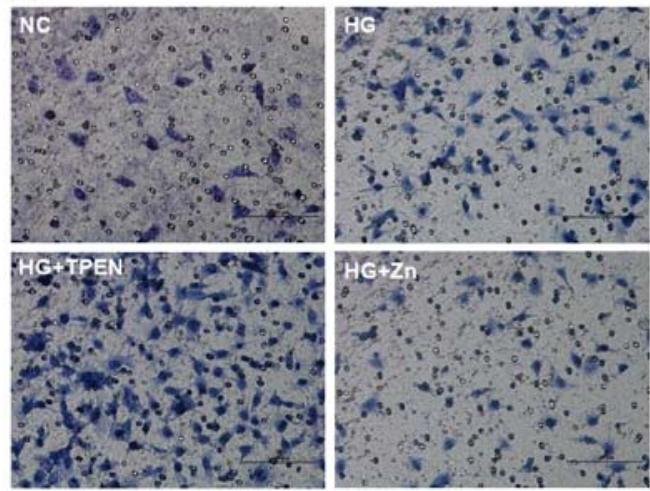

B

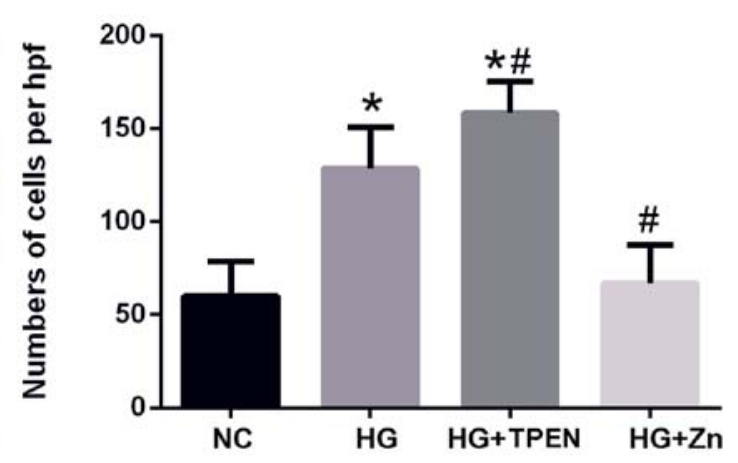

Figure 5. Effects of Zn on the HG-induced increase in the migration of HPMCs. (A and B) Transwell assay revealed the migration ability of HPMCs following co-treatment with $\mathrm{HG}$, and TPEN or $\mathrm{ZnSO}_{4}$ for $24 \mathrm{~h}$ (magnification, $\mathrm{x} 20$ ). Data are presented as the mean \pm standard deviation. P-values were determined using ANOVA with Tukey's post hoc test. "P<0.05 vs. NC; " $\mathrm{P}<0.05$ vs. HG. NC, negative control; HG, high glucose; hpf, high-power field; TPEN, N,N,N',N'-tetrakis (2-pyridylmethyl) ethylenediamine; $\mathrm{Zn}, \mathrm{ZnSO}_{4}$; $\mathrm{HPMC}$, human peritoneal mesothelial cell.

present study, the EMT markers vimentin and E-cadherin were selected for analysis. Consistent with our previous study (20), Zn supplementation significantly protected against HG-induced EMT in HPMCs. The acquisition of increased migration potential is a typical feature of cells undergoing EMT (42). Therefore, the migratory ability of cells was evaluated using a Transwell cell assay. The data revealed that the increase in migration induced by $\mathrm{HG}$ was suppressed by $\mathrm{Zn}$ supplementation.

Previous studies indicated that high concentrations of glucose induce ROS production in HPMCs $(43,44)$, which may be involved in peritoneal EMT induced by HG and glucose-based PD solution. In addition, accumulating evidence has demonstrated that $\mathrm{Zn}$ inhibition can result in oxidative stress, leading to cell component damage and alterations in cellular functions $(45,46)$. Conversely, $\mathrm{Zn}$ supplementation has been reported to decrease ROS generation $(47,48)$. In the present study, $\mathrm{Zn}$ supplementation reduced ROS generation in the HG-stimulated HPMCs, whereas $\mathrm{Zn}$ depletion increased ROS generation, indicating that $\mathrm{Zn}$ supplementation may inhibit the EMT of HPMCs by reducing ROS generation.

The Nrf2 pathway exerts an important role in antioxidative processes. Under normal conditions, Nrf2 interacts with Kelch-like ECH-associated protein 1 (Keap1) to form a compound complex in the cytoplasm, which is degraded by the ubiquitin-proteasome pathway (49). On exposure to excessive ROS, Nrf2 can dissociate from Keap1 and accumulate in the nucleus, subsequently binding with antioxidant responsive element sequences and activating antioxidant-associated genes, including NAD(P)H dehydrogenase, NQO-1 and HO-1 $(50,51)$. The present findings revealed that $\mathrm{HG}$ induced the expression of nuclear Nrf2 and the target genes of the Nrf2 pathway, indicating that HG may activate the Nrf2 pathway by inducing ROS production. Of note, $\mathrm{Zn}$ supplementation further promoted the 
A
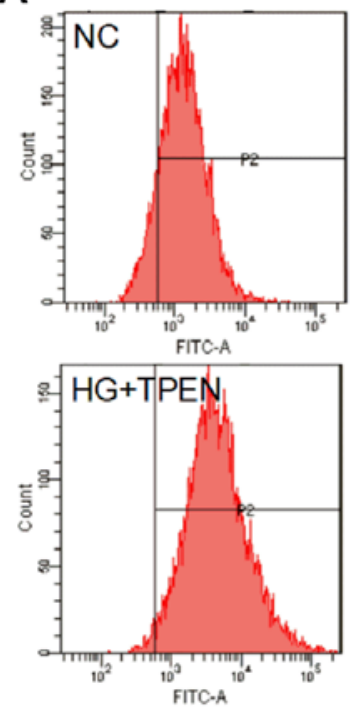

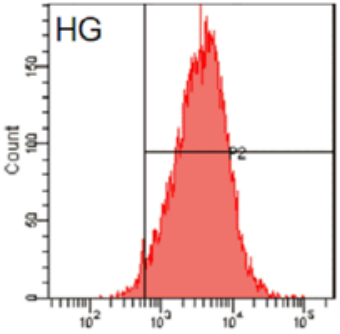
10

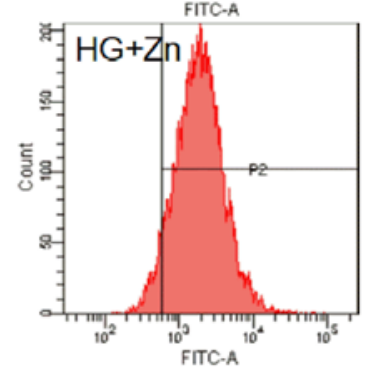

B

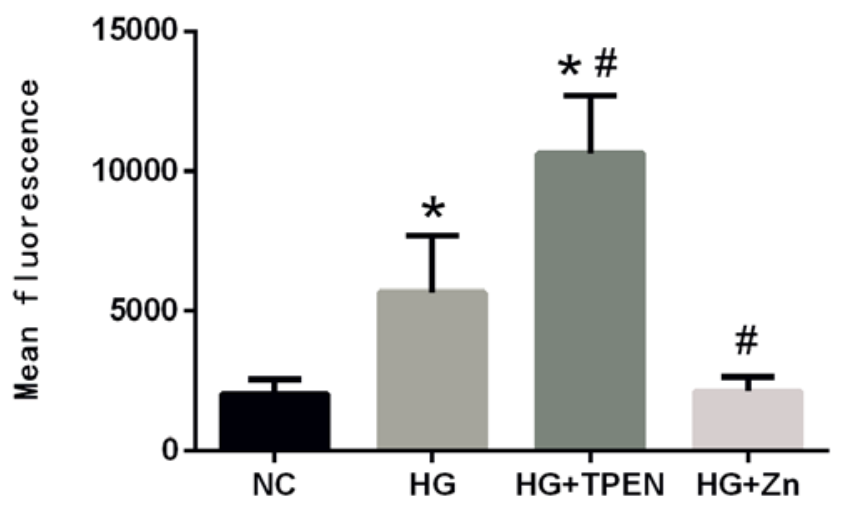

Figure 6. Effects of Zn on intracellular ROS in the HPMCs stimulated by HG. HPMCs were co-treated with HG, and TPEN or ZnSO ${ }_{4}$ for $48 \mathrm{~h}$. (A) Intracellular ROS were measured by flow cytometry using an oxidation-sensitive fluorescent probe, dichlorodihydrofluorescein diacetate, which is oxidized to 2,7'-dichlorofluorescein in the presence of ROS. (B) Data are presented as the mean \pm standard deviation. P-values were determined using ANOVA with Tukey's post hoc test. ${ }^{*} \mathrm{P}<0.05$ vs. NC; ${ }^{\prime} \mathrm{P}<0.05$ vs. HG. NC, negative control; HG, high glucose; TPEN, $\mathrm{N}, \mathrm{N}, \mathrm{N}, \mathrm{N}^{\prime}$-tetrakis (2-pyridylmethyl) ethylenediamine; $\mathrm{Zn}, \mathrm{ZnSO}_{4}$; HPMC, human peritoneal mesothelial cell; ROS, reactive oxygen species.

A

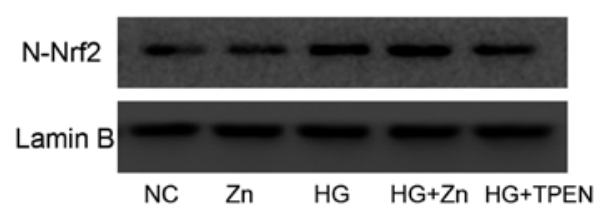

C

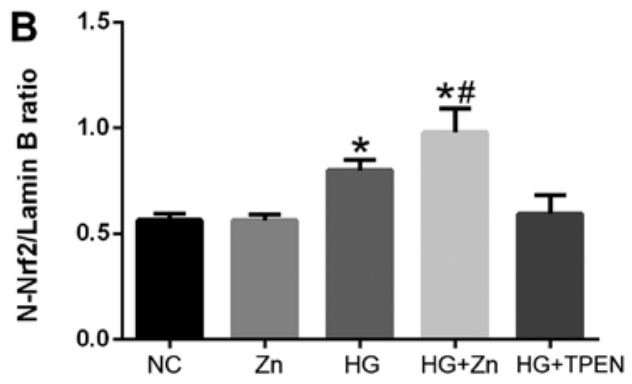

$\mathrm{HO}-1$

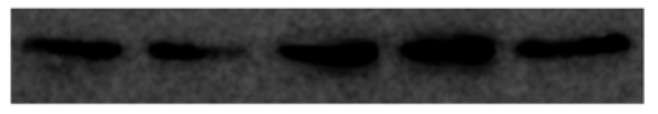

NQO1

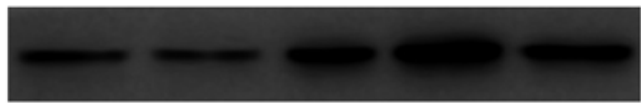

GAPDH

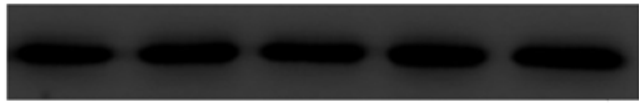

NC $\mathrm{Zn} \quad \mathrm{HG} \quad \mathrm{HG}+\mathrm{Zn}$ HG+TPEN
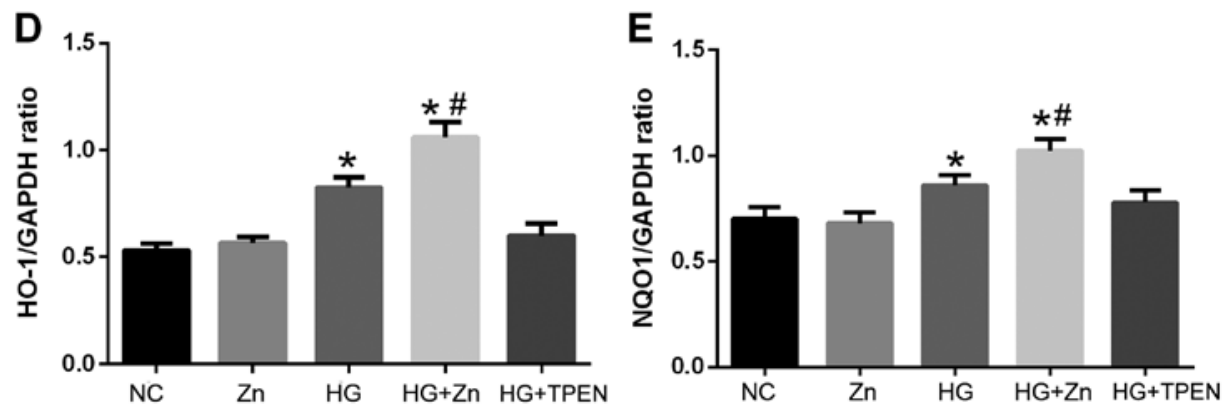

Figure 7. Effects of $\mathrm{Zn}$ on the activity of the Nrf2 antioxidant pathway in HPMCs stimulated with HG. HPMCs were co-treated with HG, and TPEN or ZnSO for $48 \mathrm{~h}$. Expression levels of (A and B) N-Nrf2, and (C-E) HO-1 and NQO1, target proteins of the Nrf2 antioxidant pathway, were analyzed via western blotting. Data are presented as the mean \pm standard deviation. P-values were determined using ANOVA with Tukey's post hoc test. ${ }^{*} \mathrm{P}<0.05$ vs. NC; ${ }^{~} \mathrm{P}<0.05$ vs. HG. $\mathrm{NC}$, negative control; HG, high glucose; TPEN, N,N,N',N'-tetrakis (2-pyridylmethyl) ethylenediamine; $\mathrm{Zn}, \mathrm{ZnSO}_{4} ; \mathrm{HPMC}$, human peritoneal mesothelial cell; $\mathrm{N}-$, nuclear; Nrf2, nuclear factor-like 2; HO-1, heme oxygenase-1; NQO1, NAD(P)H quinone dehydrogenase 1. 
expression of nuclear Nrf2 and target genes of the Nrf2 pathway, suggesting that $\mathrm{Zn}$ supplementation may inhibit the EMT of HPMCs by activating the Nrf2 pathway and subsequently decreasing oxidative stress. Kim and Vaziri (52) reported that severe oxidative stress and inflammation decreased Nrf2 activity, whereas the Nrf2 repressor, Keap1 was upregulated, and the products of Nrf2 target genes decreased, consistent with the present findings. The HG + TPEN group also demonstrated increased ROS, and a decrease in the expression of nuclear Nrf2 and target proteins of the Nrf2 pathway; however, Nrf2-associated protein expression was not significantly different compared with that in the HG group.

Our previous studies $(20,23,24)$ revealed that $\mathrm{Zn}$ attenuated the HG-induced EMT of peritoneal mesothelial cells by downregulating TGF- $\beta$ expression and inhibiting mitogen-activated protein kinase (MAPK), NF- $\kappa B$, and TGF- $\beta /$ Smad pathways. In the present study, $\mathrm{Zn}$ was reported to activate the Nrf2 pathway. Therefore, it was hypothesized that Nrf2 regulates the MAPK, NF- $\kappa B$ and TGF- $\beta / S m a d$ pathways by regulating the expression of TGF- $\beta 1$. The potential associations between these pathways will be investigated by silencing TGF- $\beta 1$ and Nrf2 in future experiments.

In conclusion, the present findings demonstrated that $\mathrm{Zn}$ reversed HG-induced EMT by activating the Nrf2 pathway. Zn supplementation may be effective in preventing HG-induced PF; however, further investigation is required to reveal whether Nrf2 pathway activation is the primary mechanism by which $\mathrm{Zn}$ alleviates PF induced by a glucose-based PD solution, and whether patients will benefit from $\mathrm{Zn}$ supplementation to prevent PF in PD.

\section{Acknowledgements}

Not applicable.

\section{Funding}

The present study was supported by the National Natural Science Foundation of China (grant no. 81370865).

\section{Availability of data and materials}

The datasets used and/or analyzed during the current study are available from the corresponding author on reasonable request.

\section{Authors' contributions}

JM designed the study. LG, YF, XZ, LY, WH, TH, ML and SD performed the experiments. LG and YF analyzed the data and prepared the manuscript. All authors read and approved the final manuscript.

\section{Ethics approval and consent to participate}

All animal experiments were approved by the Institutional Animal Ethics Committee of China Medical University.

\section{Patient consent for publication}

Not applicable.

\section{Competing interests}

The authors declare that they have no competing interests.

\section{References}

1. Prasad AS: Discovery of human zinc deficiency: Its impact on human health and diseas. Adv Nutr 4: 176-190, 2013.

2. Krediet RT and Struijk DG: Peritoneal changes in patients on long-term peritoneal dialysis. Nat Rev Nephrol 9: 419-429, 2013.

3. Yang CY, Chau YP, Chen A, Lee OK, Tarng DC and Yang AH: Targeting cannabinoid signaling for peritoneal dialysis-induced oxidative stress and fibrosis. World J Nephrol 6: 111-118, 2017.

4. Holmes CJ and Faict D: Peritoneal dialysis solution biocompatibility: Definitions and evaluation strategies. Kidney Int Suppl: S50-S56, 2003

5. Yáñez-Mó M, Lara-Pezzi E, Selgas R, Ramírez-Huesca M, Domínguez-Jiménez C, Jiménez-Heffernan JA, Aguilera A, Sánchez-Tomero JA, Bajo MA, Alvarez V, et al: Peritoneal dialysis and Epithelial-To-Mesenchymal transition of mesothelial cells. N Engl J Med 348: 403-413, 2003.

6. Wu J, Xing C, Zhang L, Mao H, Chen X, Liang M, Wang F, Ren $\mathrm{H}$, Cui H, Jiang A, et al: Autophagy promotes fibrosis and apoptosis in the peritoneum during long-term peritoneal dialysis. J Cell Mol Med 22: 1190-1201, 2018.

7. Kim YL: Update on mechanisms of ultrafiltration failure. Perit Dial Int 29 (Suppl 2): S123-S127, 2009.

8. Ha H, Yu MR and Lee HB: High glucose-induced PKC activation mediates TGF-beta 1 and fibronectin synthesis by peritoneal mesothelial cells. Kidney Int 59: 463-470, 2001.

9. Yao Q, Pawlaczyk K, Ayala ER, Styszynski A, Breborowicz A, Heimburger O, Qian JQ, Stenvinkel P, Lindholm B and Axelsson J: The role of the TGF/Smad signaling pathway in peritoneal fibrosis induced by peritoneal dialysis solutions. Nephron Exp Nephrol 109: e71-e78, 2008.

10. Ksiazek K, Breborowicz A, Jorres A and Witowski J: Oxidative stress contributes to accelerated development of the senescent phenotype in human peritoneal mesothelial cells exposed to high glucose. Free Radic Biol Med 42: 636-641, 2007.

11. Noh H, Kim JS, Han KH, Lee GT, Song JS, Chung SH, Jeon JS, Ha H and Lee HB: Oxidative stress during peritoneal dialysis: Implications in functional and structural changes in the membrane. Kidney Int 69: 2022-2028, 2006.

12. Wellinghausen $\mathbf{N}$ and Rink L: The significance of zinc for leukocyte biology. J Leukoc Biol 64: 571-577, 1998.

13. Beyersmann D and Haase H: Functions of zinc in signaling, proliferation and differentiation of mammalian cells. Biometals 14: 331-341, 2001.

14. Cousins RJ, Blanchard RK, Moore JB, Cui L, Green CL, Liuzzi JP, Cao J and Bobo JA: Regulation of zinc metabolism and genomic outcomes. J Nutr 133 (Suppl 1): 1521S-1526S, 2003.

15. Honscheid A, Rink L and Haase H: T-lymphocytes: A target for stimulatory and inhibitory effects of zinc ions. Endocr Metab Immune Disord Drug Targets 9: 132-144, 2009.

16. Takahashi M, Saito H, Higashimoto M and Hibi T: Possible inhibitory effect of oral zinc supplementation on hepatic fibrosis through downregulation of TIMP-1: A pilot study. Hepatol Res 37: 405-409, 2007.

17. Wang L, Zhou Z, Saari JT and Kang YJ: Alcohol-induced myocardial fibrosis in metallothionein-null mice: Prevention by zinc supplementation. Am J Pathol 167: 337-344, 2005.

18. Gandhi MS, Deshmukh PA, Kamalov G, Zhao T, Zhao W, Whaley JT, Tichy JR, Bhattacharya SK, Ahokas RA and Sun Y: Causes and consequences of zinc dyshomeostasis in rats with chronic aldosteronism. J Cardiovasc Pharmacol 52: 245-252, 2008.

19. Van Biervliet S, Vande Velde S, Van Biervliet JP and Robberecht E: The effect of zinc supplements in cystic fibrosis patients. Ann Nutr Metab 52: 152-156, 2008.

20. Zhang X, Wang J, Fan Y, Yang L, Wang L and Ma J: Zinc supplementation attenuates high glucose-induced epithelial-to-mesenchymal transition of peritoneal mesothelial cells. Biol Trace Elem Res 150: 229-235, 2012.

21. Duman S, Sen S, Gunal AI, Asci G, Akcicek F and Basci A: How can we standardize peritoneal thickness measurements in experimental studies in rats? Perit Dial Int 21 (Suppl 3): S338-S341, 2001.

22. Rougier JP, Moullier P, Piedagnel R and Ronco PM: Hyperosmolality suppresses but TGF beta 1 increases MMP9 in human peritoneal mesothelial cells. Kidney Int 51: 337-347, 1997. 
23. Zhang X, Liang D, Guo B, Sun L, Chi ZH, Cai Y, Wang L and Ma J: Zinc transporter 7 induced by high glucose attenuates epithelial-to-mesenchymal transition of peritoneal mesothelial cells. Biol Trace Elem Res 151: 138-147, 2013.

24. Zhang X, Liang D, Guo B, Yang L, Wang L and Ma J: Zinc inhibits high glucose-induced apoptosis in peritoneal mesothelial cells. Biol Trace Elem Res 150: 424-432, 2012.

25. Xiang S, Li M, Xie X, Xie Z, Zhou Q, Tian Y, Lin W, Zhang X, Jiang $\mathrm{H}$, Shou $\mathrm{Z}$ and Chen J: Rapamycin inhibits epithelial-to-mesenchymal transition of peritoneal mesothelium cells through regulation of Rho GTPases. FEBS J 283: 2309-2325, 2016.

26. Thiery JP and Sleeman JP: Complex networks orchestrate epithelial-mesenchymal transitions. Nat Rev Mol Cell Biol 7: 131-142, 2006.

27. Kovac S, Angelova PR, Holmstrom KM, Zhang Y, DinkovaKostova AT and Abramov AY: Nrf2 regulates ROS production by mitochondria and NADPH oxidase. Biochim Biophys Acta 1850 794-801, 2015.

28. KanlayaR,Khamchun S,Kapincharanon Cand Thongboonkerd V: Protective effect of epigallocatechin-3-gallate (EGCG) via Nrf2 pathway against oxalate-induced epithelial mesenchymal transition (EMT) of renal tubular cells. Sci Rep 6: 30233, 2016.

29. Oyama TM, Ishida S, Okano Y, Seo H and Oyama Y: Clioquinol-induced increase and decrease in the intracellular $\mathrm{Zn2+}$ level in rat thymocytes. Life Sci 91: 1216-1220, 2012

30. Priel T, Aricha-Tamir B and Sekler I: Clioquinol attenuates zinc-dependent beta-cell death and the onset of insulitis and hyperglycemia associated with experimental type I diabetes in mice. Eur J Pharmacol 565: 232-239, 2007.

31. Kim JH, Jang BG, Choi BY, Kwon LM, Sohn M, Song HK and Suh SW: Zinc chelation reduces hippocampal neurogenesis after pilocarpine-induced seizure. PLoS One 7: E48543, 2012.

32. Nitzan YB, Sekler I, Frederickson CJ, Coulter DA, Balaji RV, Liang SL, Margulis A, Hershfinkel $M$ and Silverman WF Clioquinol effects on tissue chelatable zinc in mice. J Mol Med (Berl) 81: 637-464, 2003.

33. Wang T, Wang CY, Shan ZY, Teng WP and Wang ZY: Clioquinol reduces zinc accumulation in neuritic plaques and inhibits the amyloidogenic pathway in A $\beta P P / P S 1$ transgenic mouse brain. J Alzheimers Dis 29: 549-559, 2012.

34. Cherny RA, Atwood CS, Xilinas ME, Gray DN, Jones WD, McLean CA, Barnham KJ, Volitakis I, Fraser FW, Kim Y, et al: Treatment with a copper-zinc chelator markedly and rapidly inhibits beta-amyloid accumulation in Alzheimer's disease transgenic mice. Neuron 30: 665-676, 2001.

35. Kang M, Zhao L, Ren M, Deng M and Li C: Reduced metallothionein expression induced by Zinc deficiency results in apoptosis in hepatic stellate cell line LX-2. Int J Clin Exp Med 8: 20603-20609, 2015

36. Yang H, Keen CL and Lanoue L: Influence of intracellular zinc on cultures of rat cardiac neural crest cells. Birth Defects Res B Dev Reprod Toxicol 104: 11-22, 2015.

37. Lopez-Cabrera M: Mesenchymal conversion of mesothelial cells is a key event in the pathophysiology of the peritoneum during peritoneal dialysis. Adv Med 2014: 473134, 2014.

38. Liu J, Zeng L, Zhao Y, Zhu B, Ren W and Wu C: Selenium suppresses lipopolysaccharide-induced fibrosis in peritoneal mesothelial cells through inhibition of epithelial-to-mesenchymal transition. Biol Trace Elem Res 161: 202-209, 2014.
39. Strippoli R, Moreno-Vicente R, Battistelli C, Cicchini C, Noce V, Amicone L, Marchetti A, Del Pozo MA and Tripodi M: Molecular mechanisms underlying peritoneal EMT and fibrosis. Stem Cells Int 2016: 3543678, 2016.

40. Lee JM, Dedhar S, Kalluri R and Thompson EW: The epithelial-mesenchymal transition: New insights in signaling, development, and disease. J Cell Biol 172: 973-981, 2006.

41. Kalluri R and Weinberg RA: The basics of epithelial-mesenchymal transition. J Clin Invest 119: 1420-1428, 2009.

42. Aroeira LS, Aguilera A, Sanchez-Tomero JA,Bajo MA, del Peso G, Jimenez-Heffernan JA, Selgas R and Lopez-Cabrera M: Epithelial to mesenchymal transition and peritoneal membrane failure in peritoneal dialysis patients: Pathologic significance and potential therapeutic interventions. J Am Soc Nephrol 18: 2004-2013, 2004.

43. Hsieh HL, Chi PL, Lin CC, Yang CC and Yang CM: Up-regulation of ROS-dependent matrix metalloproteinase-9 from high-glucose-challenged astrocytes contributes to the neuronal apoptosis. Mol Neurobiol 50: 520-533, 2014.

44. Lee HB, Yu MR, Song JS and Ha H: Reactive oxygen species amplify protein kinase $\mathrm{C}$ signaling in high glucose-induced fibronectin expression by human peritoneal mesothelial cells. Kidney Int 65: 1170-1179, 2004.

45. Oteiza PI, Olin KL, Fraga CG and Keen CL: Zinc deficiency causes oxidative damage to proteins, lipids and DNA in rat testes. J Nutr 125: 823-829, 1995.

46. Ho E and Ames BN: Low intracellular zinc induces oxidative DNA damage, disrupts p53, NFkappa B, and AP1 DNA binding, and affects DNA repair in a rat glioma cell line. Proc Natl Acad Sci USA 99: 16770-16775, 2002.

47. Prasad AS: Clinical, immunological, anti-inflammatory and antioxidant roles of zinc. Exp Gerontol 43: 370-377, 2008.

48. Bao B, Prasad AS, Beck FW, Snell D, Suneja A, Sarkar FH, Doshi N, Fitzgerald JT and Swerdlow P: Zinc supplementation decreases oxidative stress, incidence of infection, and generation of inflammatory cytokines in sickle cell disease patients. Transl Res 152: 67-80, 2008

49. Taguchi K, Motohashi $\mathrm{H}$ and Yamamoto M: Molecular mechanisms of the Keap1-Nrf2 pathway in stress response and cancer evolution. Genes Cells 16: 123-140, 2011.

50. McMahon M, Lamont DJ, Beattie KA and Hayes JD: Keap1 perceives stress via three sensors for the endogenous signaling molecules nitric oxide, zinc, and alkenals. Proc Natl Acad Sci USA 107: 18838-18843, 2010

51. Wang R, Paul VJ and Luesch H: Seaweed extracts and unsaturated fatty acid constituents from the green alga Ulva lactuca as activators of the cytoprotective Nrf2-ARE pathway. Free Radic Biol Med 57: 141-153, 2013.

52. Kim HJ and Vaziri ND: Contribution of impaired Nrf2-Keap1 pathway to oxidative stress and inflammation in chronic renal failure. Am J Physiol Renal Physiol 298: F662-F671, 2010.

This work is licensed under a Creative Commons Attribution-NonCommercial-NoDerivatives 4.0 International (CC BY-NC-ND 4.0) License. 